\title{
Common Weak Linear Copositive Lyapunov Functions for Positive Switched Linear Systems
}

\author{
Yuangong Sun $\mathbb{D}^{1},{ }^{1,2}$ Zhaorong $\mathrm{Wu},{ }^{1}$ and Fanwei Meng ${ }^{3}$ \\ ${ }^{1}$ School of Mathematical Sciences, University of Jinan, Jinan, Shandong 250022, China \\ ${ }^{2}$ College of Mathematics and Systems Science, Shandong University of Science and Technology, Qingdao, Shandong 266590, China \\ ${ }^{3}$ School of Mathematical Sciences, Qufu Normal University, Qufu, Shandong 273165, China \\ Correspondence should be addressed to Yuangong Sun; sunyuangong@163.com
}

Received 7 November 2017; Accepted 31 January 2018; Published 26 February 2018

Academic Editor: Arturo Buscarino

Copyright (C) 2018 Yuangong Sun et al. This is an open access article distributed under the Creative Commons Attribution License, which permits unrestricted use, distribution, and reproduction in any medium, provided the original work is properly cited.

\begin{abstract}
Lyapunov functions play a key role in the stability analysis of complex systems. In this paper, we study the existence of a class of common weak linear copositive Lyapunov functions (CWCLFs) for positive switched linear systems (PSLSs) which generalize the conventional common linear copositive Lyapunov functions (CLCLFs) and can be used as handy tool to deal with the stability of PSLSs not covered by CLCLFs. We not only establish necessary and sufficient conditions for the existence of CWCLFs but also clearly describe the algebraic structure of all CWCLFs. Numerical examples are also given to demonstrate the effectiveness of the obtained results.
\end{abstract}

\section{Introduction}

Positive dynamical system is one for which nonnegative initial conditions give rise to nonnegative trajectories [1-3]. In recent years, stability issue for PSLS has been addressed for both practical applications in cooperative control of multiagent systems [4-8] and for theoretical reasons in [9-17] to name a few. For PSLSs, linear copositive Lyapunov functions play an important role in the stability analysis. It is well known that the existence of CLCLFs implies asymptotic stability of PSLSs under arbitrary switching. Moreover, necessary and sufficient conditions for the existence of CLCLFs have been extensively investigated in [18-22].

Since the existence of CLCLFs is only a sufficient condition for asymptotic stability of PSLSs under arbitrary switching [18], it is necessary and significant to study asymptotic stability of the PSLS when it does not have a CLCLF. Motivated by the idea in $[23,24]$, where common joint quadratic Lyapunov functions were introduced for the first time, a class of common joint linear copositive Lyapunov functions (CJCLFs) were proposed to design time-dependent switching signals under which the PSLS is asymptotically stable [25, 26]. Moreover, such a method in [26] has been successfully applied to consensus of multiagent systems.
Notice that CJCLFs play an important role in the stability analysis of the PSLS. It is necessary to make it clear whether the PSLS has a CJCLF. So far, the existence of CJCLFs is still untouched except for the simpler cases $n=2$ and $n=3$ in [27]. Unlike CLCLFs, CJCLFs are determined by a series of nonstrict inequalities on each individual system combined with a strict inequality satisfied jointly, which leads to some difficulty in the analysis of the existence of CJCLFs.

In order to better solve the existence of CJCLFs, we will first introduce a class of common weak linear copositive Lyapunov functions (CWCLFs) determined only by a series of nonstrict inequalities on each individual system. By using matrix theory, necessary and sufficient conditions for the existence of CWCLFs have been established. What is more, the algebraic structure of all CWCLFs for PSLSs has been portrayed clearly. Consequently, the existence of CJCLFs becomes easily verifiable based on the algebraic structure of CWCLFs.

The paper is organized as follows. In Section 2, we will present the notations used throughout this paper as well as some preliminary results that are used later. Section 3 then focuses on deriving necessary and sufficient conditions for the existence of CWCLFs for PSLSs. In Section 4, we give two examples to demonstrate the effectiveness of the 
obtained theoretical results. Finally, conclusions are drawn in Section 5.

\section{Problem Statement and Preliminaries}

Throughout this paper, $\langle m\rangle$ is the set of integers $\{1,2, \ldots, m\}$ for any positive integer $m$. If all entries of vector $x$ are positive (nonpositive, negative), we denote $x>0(\preceq 0, \prec 0)$. For a matrix $A$, denote $A \preceq 0$ if all its entries are nonpositive. Denote the $j$-th column and the $(i, j)$-th component of matrix $A_{k}$ by $\operatorname{col}_{j}\left(A_{k}\right)$ and $a_{i j}^{(k)}$, respectively. $I_{n}$ is an $n$-dimensional identity matrix. A Metzler matrix is a real square matrix, whose off-diagonal entries are nonnegative. A square matrix is Hurwitz if the real part of each of its eigenvalues is negative.

Consider the following continuous-time switched linear system:

$$
\dot{x}(t)=A_{\sigma(t)} x(t), \quad t \geq 0,
$$

where $x$ is the $n$-dimensional state vector, the piecewise continuous function $\sigma:[0,+\infty) \rightarrow\langle m\rangle$ is the switching signal, and $A_{k}$ is an $n \times n$-matrix for each $k \in\langle m\rangle$.

As usual, system (1) is said to be positive, if $x(t) \geq 0$ for any $t \geq 0$, any $x(0) \geq 0$, and arbitrary switching [12]. We know that system (1) is positive if and only if $A_{k}$ is a Metzler matrix for each $k \in\langle m\rangle$. A CLCLF method is usually used for asymptotic stability of PSLS (1) under arbitrary switching. Given an $n$-dimensional vector $v>0, V(x)=v^{T} x$ (or briefly $v$ ) is said to be a CLCLF of PSLS (1) (or the family of Metzler matrices $\left.\mathscr{A}=\left\{A_{1}, A_{2}, \ldots, A_{m}\right\}\right)$ if

$$
v^{T} A_{k} \prec 0, \quad k \in\langle m\rangle .
$$

Note that (2) is only a sufficient condition for asymptotic stability of PSLS (1) under arbitrary switching. There are obviously many examples where such a sufficient condition does not hold even if PSLS (1) is asymptotically stable under arbitrary switching. Therefore, we consider the following weaker condition:

$$
v^{T} A_{k} \preceq 0, \quad k \in\langle m\rangle .
$$

In order to guarantee asymptotic stability of PSLS (1) under appropriately chosen switching signals, CJCLFs were proposed in [27]. Given an $n$-dimensional vector $v>0$, $V(x)=v^{T} x$ is said to be a CJCLF of PSLS (1) if (3) holds and

$$
v^{T} \sum_{k=1}^{m} A_{k} \prec 0
$$

For the case $m=2$, it was shown in [25] that PSLS (1) is asymptotically stable under arbitrary switching if it has a CJCLF. Therefore, CJCLFs play an important role in the analysis for asymptotic stability of PSLS (1).

For particular cases $n=2$ and $n=3$, the existence of CJCLFs of PSLS (1) has been studied in [27]. For the general case, it remains unexplored so far. In this paper, we will introduce the definition of CWCLFs. Given an $n$-dimensional vector $v>0, V(x)=v^{T} x$ (or briefly $v$ ) is said to be a CWCLF of PSLS (1) (or $\mathscr{A}$ ) if (3) holds. If the algebraic structure of all CWCLFs can be clearly described, condition (4) becomes easily verifiable, and hence the existence of CJCLFs can be solved accordingly.

Under the assumption that there exists a CWCLF of $\mathscr{A}$, we have (H1): $a_{j j}^{(k)} \leq 0$ for any $j \in\langle n\rangle$ and $k \in\langle m\rangle ; a_{i j}^{(k)}=0$ for all $i \in\langle n\rangle$ if $a_{j j}^{(k)}=0$ for some $j \in\langle n\rangle$ and $k \in\langle m\rangle$. In the following, it is always assumed that (H1) holds. Otherwise, $\mathscr{A}$ does not have a CWCLF.

Note that $\mathscr{A}$ has a CWCLF if and only if the family of Metzler matrices $\left\{A_{k} D_{k}^{-1}: k \in\langle m\rangle\right\}$ has a CWCLF, where $D_{k}=\operatorname{diag}\left\{d_{1}^{(k)}, d_{2}^{(k)}, \ldots, d_{n}^{(k)}\right\}$ is a diagonal matrix with

$$
d_{i}^{(k)}= \begin{cases}-a_{i i}^{(k)}, & \text { if } a_{i i}^{(k)}<0, \\ 1, & \text { if } a_{i i}^{(k)}=0 .\end{cases}
$$

For the sake of convenience, assume throughout this paper that $a_{i i}^{(k)}=-1(0)$ for $i \in\langle n\rangle$ and $k \in\langle m\rangle$.

In the sequel, we define a sequence of positive integers (SPI) $\left\{j_{1}, j_{2}, \ldots, j_{p}\right\}$ for $p \in\langle n\rangle$ such that $1 \leq j_{1}<j_{2}<\cdots<$ $j_{p} \leq n$. Denote the $n \times p$-matrix:

$$
\begin{aligned}
& A_{k_{j_{1}} k_{j_{2}} \cdots k_{j_{p}}} \\
& \quad=\left[\operatorname{col}_{j_{1}}\left(A_{k_{j_{1}}}\right), \operatorname{col}_{j_{2}}\left(A_{k_{j_{2}}}\right), \ldots, \operatorname{col}_{j_{p}}\left(A_{k_{j_{p}}}\right)\right],
\end{aligned}
$$

where $k_{j_{i}} \in \Lambda_{j_{i}}$ for $i \in\langle p\rangle$ and the nonempty index set $\Lambda_{i}=$ $\left\{k \in\langle m\rangle: a_{i i}^{(k)}<0\right\}$ for $i \in\langle n\rangle$. Let

$$
\mathscr{L}_{j_{1} j_{2} \cdots j_{p}}=\left\{\widetilde{A}_{k_{j_{1}} k_{j_{2}} \cdots k_{j_{p}}}: k_{j_{i}} \in \Lambda_{j_{i}}, i \in\langle p\rangle\right\},
$$

where $\widetilde{A}_{k_{j_{1}} k_{j_{2}} \cdots k_{j_{p}}}$ is a $p \times p$-matrix obtained from $A_{k_{j_{1}} k_{j_{2}} \cdots k_{j_{p}}}$ by deleting all its rows except those labelled by $j_{1}, j_{2}, \ldots, j_{p}$.

Remark 1. It follows from assumption (H1) that $\mathscr{A}$ has a CWCLF if and only if $\mathscr{L}_{12 \cdots n}$ has a CWCLF. Moreover, for any SPI $\left\{j_{1}, j_{2}, \ldots, j_{p}\right\}$ and any $p \in\langle n\rangle, \mathscr{L}_{j_{1} j_{2} \cdots j_{p}}$ has a CWCLF if $\mathscr{L}_{12 \cdots n}$ has a CWCLF.

For $p \in\langle n-1\rangle(n \geq 2)$, decompose the matrix $\widetilde{A}_{k_{j_{1}} k_{j_{2}} \cdots k_{j_{p+1}}}$ as follows:

$$
\widetilde{A}_{k_{j_{1}} k_{j_{2}} \cdots k_{j_{p+1}}}=\left(\begin{array}{cc}
\widetilde{A}_{k_{j_{1}} k_{j_{2}} \cdots k_{j_{p}}} & \xi_{k_{j_{p+1}}} \\
\eta_{k_{j_{p+1}}}^{T} & -1
\end{array}\right)
$$

where $\xi_{k_{j_{p+1}}}$ and $\eta_{k_{j_{p+1}}}$ are the corresponding $p$-dimensional column vectors; $k_{j_{i}} \in \Lambda_{j_{i}}$ for $i \in\langle p+1\rangle$. If the matrix $\widetilde{A}_{k_{j_{1}} k_{j_{2}} \cdots k_{j_{p}}}$ is invertible, the equation

$$
y^{T} \widetilde{A}_{k_{j_{1}} k_{j_{2}} \cdots k_{j_{p}}}=-\eta_{k_{j_{p+1}}}^{T}
$$

has a unique solution, where $y$ is a $p$-dimensional column vector. We denote the solution of (9) by $\theta_{k_{j_{1}} k_{j_{2}} \cdots k_{j_{p+1}}}$ when it has a unique solution. Let

$$
\Theta_{j_{1} j_{2} \cdots j_{p+1}}=\left\{\theta_{k_{j_{1}} k_{j_{2}} \cdots k_{j_{p+1}}}: k_{j_{i}} \in \Lambda_{j_{i}}, i \in\langle p+1\rangle\right\} .
$$


We now introduce several lemmas required in the proof of the main results. Since new notations are introduced in this paper, the following lemmas in $\mathrm{Wu}$ and Sun (2013) are rewritten appropriately.

Lemma 2 (see [22]). Given an SPI $\left\{j_{1}, j_{2}, \ldots, j_{p+1}\right\}$ and $p \in$ $\langle n-1\rangle(n \geq 2)$, if $\mathscr{L}_{j_{1} j_{2} \cdots j_{p}}$ has a CLCLF, then there exists a $p+1$-tuple $\left(\bar{k}_{j_{1}}, \bar{k}_{j_{2}}, \ldots, \bar{k}_{j_{p+1}}\right), \bar{k}_{j_{i}} \in \Lambda_{j_{i}}, i \in\langle p+1\rangle$, such that

$$
\begin{aligned}
\theta_{\bar{k}_{j_{1}}}^{T} \bar{k}_{j_{2}} \cdots \bar{k}_{j_{p+1}} \widetilde{A}_{k_{j_{1}} k_{j_{2}} \cdots k_{j_{p}}} & \leq-\eta_{k_{j_{p+1}}}^{T}, \\
\theta_{k_{j_{1}} k_{j_{2}} \cdots k_{j_{p+1}}} & \leq \theta_{\bar{k}_{j_{1}} \bar{k}_{j_{2}} \cdots \bar{k}_{j_{p+1}}},
\end{aligned}
$$

where $k_{j_{i}} \in \Lambda_{j_{i}}, i \in\langle p+1\rangle$.

For $p=1$, noting that $\widetilde{A}_{k_{j_{1}}}=-1$ for $k_{j_{1}} \in \Lambda_{j_{1}}, \mathscr{L}_{j_{1}}=$ $\{-1\}$ for any $j_{1} \in\langle n\rangle$; it is obvious that $\mathscr{L}_{j_{1}}$ has a CLCLF. By Lemma 2, let

$$
\begin{aligned}
\theta_{j_{1} j_{2}} & =\theta_{\bar{k}_{j_{1}} \bar{k}_{j_{2}}}, \\
\lambda_{j_{1} j_{2}} & =\max \left\{\theta_{j_{1} j_{2}}^{T} \xi_{j_{j_{2}}}: k_{j_{2}} \in \Lambda_{j_{2}}\right\} .
\end{aligned}
$$

That is, $\theta_{j_{1} j_{2}}$ and $\lambda_{j_{1} j_{2}}$ are always well defined.

Generally speaking, given an SPI $\left\{j_{1}, j_{2}, \ldots, j_{p+1}\right\}$ and $p \in$ $\langle n-1\rangle$, if $\mathscr{L}_{j_{1} j_{2} \cdots j_{p}}$ has a CLCLF, by Lemma 2, we can define

$$
\begin{aligned}
\theta_{j_{1} j_{2} \cdots j_{p+1}} & =\theta_{\bar{k}_{j_{1}} \bar{k}_{j_{2}} \cdots \bar{k}_{j_{p+1}}}, \\
\lambda_{j_{1} j_{2} \cdots j_{p+1}} & =\max \left\{\theta_{j_{1} j_{2} \cdots j_{p+1}}^{T} \xi_{k_{j_{p+1}}}: k_{j_{p+1}} \in \Lambda_{j_{p+1}}\right\} .
\end{aligned}
$$

Lemma 3 (see [22]). Given an SPI $\left\{j_{1}, j_{2}, \ldots, j_{p+1}\right\}$ and $p \in$ $\langle n-1\rangle(n \geq 2), \mathscr{L}_{j_{1} j_{2} \cdots j_{p+1}}$ has a CLCLF if and only if $\lambda_{j_{1} j_{2}}<$ $1, \lambda_{j_{1} j_{2} j_{3}}<1, \ldots, \lambda_{j_{1} j_{2} \cdots j_{p+1}}<1$.

Lemma 4 (see [28]). For an $n \times n$-Metzler matrix $A$, if $A$ is Hurwitz, then $A^{-1} \leq 0$.

\section{Main Results}

We first present the following lemma which plays a key role in the proof of the main results.

Lemma 5. Given $p \in\langle n-1\rangle(n \geq 2)$, assume that $\mathscr{L}_{j_{1} j_{2} \cdots j_{p}}$ has a CLCLF for any SPI $\left\{j_{1}, j_{2}, \ldots, j_{p}\right\}$. Suppose also that $\mathscr{L}_{j_{1} j_{2} \cdots j_{p+1}}$ has a CWCLF for some SPI $\left\{j_{1}, j_{2}, \ldots, j_{p+1}\right\}$; then $\lambda_{j_{1} j_{2} \cdots j_{p+1}} \leq 1$, and all CWCLFs of $\mathscr{L}_{j_{1} j_{2} \cdots j_{p+1}}$ have the form $\mu\left(\theta_{j_{1} j_{2} \cdots j_{p+1}}^{T}, 1\right)^{T}$ when $\lambda_{j_{1} j_{2} \cdots j_{p+1}}=1$, where $\mu>0$ is a constant.

Proof. Since $\mathscr{L}_{j_{1} j_{2} \ldots j_{p}}$ has a CLCLF, by using Lemma 2, we see that $\theta_{j_{1} j_{2} \cdots j_{p+1}}$ and $\lambda_{j_{1} j_{2} \cdots j_{p+1}}$ are well defined by (14) and (15) for the given SPI $\left\{j_{1}, j_{2}, \ldots, j_{p+1}\right\}$. Suppose that $v>0$ is a CWCLF of $\mathscr{L}_{j_{1} j_{2} \cdots j_{p+1}}$. Set $v=\mu\left(u^{T}, 1\right)^{T}$, where $u>0$ is a $p$-dimensional column vector and $\mu>0$ is an appropriate constant. It is obvious that $\left(u^{T}, 1\right)^{T}$ is also a CWCLF of $\mathscr{L}_{j_{1} j_{2} \cdots j_{p+1}}$. Consequently, we get from (8) that

$$
\begin{aligned}
& u^{T} \widetilde{A}_{k_{j_{1}} k_{j_{2}} \cdots k_{j_{p}}} \preceq-\eta_{k_{j_{p+1}}}^{T}, \quad \forall k_{j_{i}} \in \Lambda_{j_{i}}, i \in\langle p+1\rangle, \\
& u^{T} \xi_{k_{j_{p+1}}} \leq 1, \quad \forall k_{j_{p+1}} \in \Lambda_{j_{p+1}} .
\end{aligned}
$$

On the other hand, by the definition of $\theta_{j_{1} j_{2} \cdots j_{p+1}}$, we have that

$$
\theta_{j_{1} j_{2} \cdots j_{p+1}}^{T} \widetilde{A}_{\bar{k}_{j_{1}} \bar{k}_{j_{2}} \cdots \bar{k}_{j_{p}}}=-\eta_{\bar{k}_{j_{p+1}}}^{T},
$$

where $\bar{k}_{j_{i}} \in \Lambda_{j_{i}}, i \in\langle p+1\rangle$, are defined as in Lemma 2. This together with (16) yields that

$$
\left[u-\theta_{j_{1} j_{2} \cdots j_{p+1}}\right]^{T} \widetilde{A}_{\bar{k}_{j_{1}} \bar{k}_{j_{2}} \ldots \bar{k}_{j_{p}}} \leq 0 .
$$

Noting that $\widetilde{A}_{\bar{k}_{j_{1}} \bar{k}_{j_{2}} \cdots \bar{k}_{j_{p}}}$ is Hurwitz since $\mathscr{L}_{j_{1} j_{2} \cdots j_{p}}$ has a CLCLF, it follows from Lemma 4 and (19) that

$$
u \geq \theta_{j_{1} j_{2} \cdots j_{p+1}} .
$$

Substituting (20) into (17) gives

$$
\theta_{j_{1} j_{2} \cdots j_{p+1}}^{T} \xi_{k_{j_{p+1}}} \leq u^{T} \xi_{k_{j_{p+1}}} \leq 1, \quad \forall k_{j_{p+1}} \in \Lambda_{j_{p+1}} .
$$

It implies that $\lambda_{j_{1} j_{2} \cdots j_{p+1}} \leq 1$.

Next, we show that $u=\theta_{j_{1} j_{2} \cdots j_{p+1}}$ if $\lambda_{j_{1} j_{2} \cdots j_{p+1}}=1$. By the definition of $\lambda_{j_{1} j_{2} \cdots j_{p+1}}$, there exists an index $\bar{k}_{j_{p+1}} \in \Lambda_{j_{p+1}}$ such that

$$
\theta_{j_{1} j_{2} \cdots j_{p+1}}^{T} \xi_{\bar{k}_{j_{p+1}}}=1
$$

From (21) and (22), we have

$$
\left[u-\theta_{j_{1} j_{2} \cdots j_{p+1}}\right]^{T} \xi_{\bar{k}_{j_{p+1}}}=0 .
$$

If $\xi_{\bar{k}_{p_{p+1}}}>0$, we can directly conclude that $u=\theta_{j_{1} j_{2} \cdots j_{p+1}}$ from (20) and (23). Otherwise, $\xi_{\bar{k}_{j_{p+1}}}$ has at least one zero entry. For the sake of convenience, assume that the last component of $\xi_{\bar{k}_{j_{p+1}}}$ is zero, and all the others are positive. That is,

$$
\xi_{\bar{k}_{j_{p+1}}}=\left(\tilde{\xi}_{\bar{k}_{j_{p+1}}^{T}}, 0\right)^{T},
$$

where $\widetilde{\xi}_{\bar{k}_{j_{p+1}}}>0$ is a $(p-1)$-dimensional column vector. Set

$$
\begin{aligned}
u & =\left[\widetilde{u}^{T}, \widehat{u}\right]^{T}, \\
\theta_{j_{1} j_{2} \cdots j_{p+1}} & =\left[\widetilde{\theta}_{j_{1} j_{2} \cdots j_{p+1}}^{T}, \widehat{\theta}_{j_{1} j_{2} \cdots j_{p+1}}\right]^{T},
\end{aligned}
$$

where $\tilde{u}$ and $\widetilde{\theta}_{j_{1} j_{2} \cdots j_{p+1}}$ are the corresponding $(p-1)$ dimensional column vectors; $\widehat{u}$ and $\widehat{\theta}_{j_{1} j_{2} \cdots j_{p+1}}$ are appropriate constants. From (22)-(25), we obtain

$$
\begin{aligned}
\widetilde{\theta}_{j_{1} j_{2} \cdots j_{p+1}}^{T} \widetilde{\xi}_{\bar{k}_{j_{p+1}}} & =1, \\
{\left[\widetilde{u}-\widetilde{\theta}_{j_{1} j_{2} \cdots j_{p+1}}\right]^{T} \widetilde{\xi}_{\bar{k}_{j_{p+1}}} } & =0 .
\end{aligned}
$$


Since $\widetilde{\xi}_{\bar{k}_{j_{p+1}}}>0$, we can get from (20) and (27) that

$$
\widetilde{u}=\widetilde{\theta}_{j_{1} j_{2} \cdots j_{p+1}} .
$$

Now, it is sufficient to show that $\widehat{u}=\widehat{\theta}_{j_{1} j_{2} \cdots j_{p+1}}$. Otherwise, $\widehat{u}>$ $\widehat{\theta}_{j_{1} j_{2} \cdots j_{p+1}}$ from (20). We now decompose the $(p+1) \times(p+1)$ matrix $\widetilde{A}_{\bar{k}_{j_{1}} \bar{k}_{j_{2}} \cdots \bar{k}_{j_{p+1}}}$ into the following form:

$$
\left(\begin{array}{ccc}
\widetilde{A}_{\bar{k}_{j_{1}} \bar{k}_{j_{2}} \cdots \bar{k}_{j_{p-1}}} & \xi_{\bar{k}_{j_{p}}} & \widetilde{\xi}_{\bar{k}_{j_{p+1}}} \\
\eta_{\bar{k}_{j_{p}}}^{T} & -1 & 0 \\
\widetilde{\eta}_{\bar{k}_{j_{p+1}}}^{T} & \bar{\eta}_{\bar{k}_{j_{p+1}}} & -1
\end{array}\right) .
$$

From (19), (25), and (28), we have

$$
\left[\widehat{u}-\widehat{\theta}_{j_{1} j_{2} \cdots j_{p+1}}\right] \eta_{\bar{k}_{j_{p}}}^{T} \preceq 0 .
$$

Since $\widehat{u}>\widehat{\theta}_{j_{1} j_{2} \cdots j_{p+1}}$, the above inequality implies that $\eta_{\bar{k}_{j_{p}}}$ is a zero vector. Based on (9) and (25), a straightforward computation yields that

$$
\widetilde{\theta}_{j_{1} j_{2} \cdots j_{p+1}}^{T} \widetilde{A}_{\bar{k}_{j_{1}} \bar{k}_{j_{2}} \ldots \bar{k}_{j_{p-1}}}=-\widetilde{\eta}_{\bar{k}_{j_{p+1}}^{T}}^{T} .
$$

It implies that $\widetilde{\theta}_{j_{1} j_{2} \cdots j_{p+1}} \in \Theta_{j_{1} j_{2} \cdots j_{p-1} j_{p+1}}$. From (12), (14), (15), and (26), we get

$$
\lambda_{j_{1} j_{2} \cdots j_{p-1} j_{p+1}} \geq \widetilde{\theta}_{j_{1} j_{2} \cdots j_{p+1}}^{T} \widetilde{\xi}_{\bar{k}_{j_{p+1}}}=1,
$$

which is a contradiction with the fact that $\mathscr{L}_{j_{1} j_{2} \cdots j_{p-1} j_{p+1}}$ has a CLCLF. Consequently, $u=\theta_{j_{1} j_{2} \cdots j_{p+1}}$ when $\lambda_{j_{1} j_{2} \cdots j_{p+1}}=1$; that is, all CWCLFs of $\mathscr{L}_{j_{1} j_{2} \cdots j_{p+1}}$ have the form $\mu\left(\theta_{j_{1} j_{2} \cdots j_{p+1}}, 1\right)^{T}$. This completes the proof of Lemma 5.

Remark 6. Assume that the family of Metzler matrices $\mathscr{A}$ has a CWCLF. Noting that there is always a CLCLF of $\mathscr{L}_{j_{1}}$ for any $j_{1} \in\langle n\rangle$, we get from Lemma 5 that $\lambda_{j_{1} j_{2}} \leq 1$ for any SPI $\left\{j_{1}, j_{2}\right\}$. If $\lambda_{j_{1} j_{2}}<1$ for any SPI $\left\{j_{1}, j_{2}\right\}$, Based on Lemma 3 , $\mathscr{L}_{j_{1} j_{2}}$ has a CLCLF for any SPI $\left\{j_{1}, j_{2}\right\}$. By using Lemma 5 again, we further get $\lambda_{j_{1} j_{2} j_{3}} \leq 1$ for any SPI $\left\{j_{1}, j_{2}, j_{3}\right\}$. Therefore, the existence of CWCLFs of $\mathscr{A}$ implies that there is at least one $p \in\{2,3, \ldots, n\}(n \geq 2)$ such that $\lambda_{j_{1} j_{2} \cdots j_{q}}<1$ for any SPI $\left\{j_{1}, j_{2}, \ldots, j_{q}\right\}$ and any $q \in\langle p-1\rangle$. For the trivial case $q=1$, we denote $\lambda_{j_{1}}=0$.

We first establish a result for the case when $p=n$ in Remark 6. That means (H2) $\lambda_{j_{1} j_{2} \cdots j_{q}}<1$ for any SPI $\left\{j_{1}, j_{2}, \ldots, j_{q}\right\}$ and any $q \in\langle n-1\rangle(n \geq 2)$.

Theorem 7. Assume that (H2) holds. There exists a CWCLF of $\mathscr{A}$ if and only if $\lambda_{12 \cdots n} \leq 1$ and $\theta_{12 \cdots n}>0$ when $\lambda_{12 \cdots n}=1$. Moreover, all CWCLFs of $\mathscr{A}$ are the same as CLCLFs if $\lambda_{12 \cdots n}<$ 1 , and all CWCLFs of $\mathscr{A}$ have the form $\mu\left(\theta_{12 \cdots n}^{T}, 1\right)^{T}$ if $\lambda_{12 \cdots n}=$ 1 , where $\mu>0$ is a constant.
Proof.

Necessity. We first get from (H2) and Lemma 3 that $\mathscr{L}_{j_{1} j_{2} \cdots j_{q}}$ has a CLCLF for any SPI $\left\{j_{1}, j_{2}, \ldots, j_{q}\right\}$ and any $q \in\langle n-1\rangle$. By using Lemma 5, we have that $\lambda_{12 \cdots n} \leq 1$. If $\lambda_{12 \cdots n}<1$, we conclude from Lemma 3 that $\mathscr{A}$ has a CLCLF. Therefore, all CWCLFs of $\mathscr{A}$ are the same as CLCLFs. If $\lambda_{12 \cdots n}=1$, from Lemma 5 , we see that all CWCLFs of $\mathscr{A}$ have the form $\mu\left(\theta_{12 \cdots n}^{T}, 1\right)^{T}$, and hence $\theta_{12 \cdots n}>0$.

Sufficiency. If $\lambda_{12 \cdots n}<1$, we get from (H2) and Lemma 3 that there is a CLCLF of $\mathscr{A}$, which is also a CWCLF of $\mathscr{A}$. If $\lambda_{12 \cdots n}=$ 1 , we get from (11), (14), and (15) that $\left(\theta_{12 \cdots n}^{T}, 1\right)^{T}$ is a CWCLF of $\mathscr{A}$ since $\theta_{12 \cdots n}>0$. This completes the proof of Theorem 7 .

For the particular case when $a_{n i}^{(k)}>0$ for $i \in\langle n-1\rangle$ and $k \in\langle m\rangle$, if there is a CWCLF of $\mathscr{A}$, then there is a CLCLF of $\mathscr{L}_{12 \cdots n-1}$, and hence $\lambda_{12}<1, \lambda_{123}<1, \ldots, \lambda_{12 \cdots n-1}<1$ by Lemma 3. Suppose also that $a_{i n}^{(k)}>0$ for $i \in\langle n-1\rangle$ and $k \in\langle m\rangle$. Following the proof of Lemma 5, we see that Lemma 5 holds true under the assumption that $\mathscr{L}_{12 \cdots n-1}$ has a CLCLF. Similar to the proof of Theorem 7, we have the following corollary.

Corollary 8. Assume that $a_{n i}^{(k)}>0$ and $a_{i n}^{(k)}>0$ for $i \in\langle n-1\rangle$ and $k \in\langle m\rangle$. There exists a CWCLF of $\mathscr{A}$ if and only if $\lambda_{12}<1$, $\lambda_{123}<1, \ldots, \lambda_{12 \cdots n-1}<1, \lambda_{12 \cdots n} \leq 1$, and $\theta_{12 \cdots n}>0$ when $\lambda_{12 \cdots n}=1$. Moreover, all CWCLFs of $\mathscr{A}$ are the same as CLCLFs if $\lambda_{12 \cdots n}<1$, and all CWCLFs of $\mathscr{A}$ have the form $\mu\left(\theta_{12 \cdots n}^{T}, 1\right)^{T}$ if $\lambda_{12 \cdots n}=1$, where $\mu>0$ is a constant.

Next, we consider the case when the assumption in Theorem 7 does not hold. By Remark 6, we have that (H3) there exists an integer $p \in\langle n-2\rangle$ with $n \geq 3$ such that $\lambda_{j_{1} j_{2} \cdots j_{q}}<1$ for any SPI $\left\{j_{1}, j_{2}, \ldots, j_{q}\right\}$ and any $q \in\langle p\rangle$. In addition, there exists an SPI $\left\{\bar{j}_{1}, \bar{j}_{2}, \ldots, \bar{j}_{p+1}\right\}$ such that $\lambda_{\bar{j}_{1} \bar{j}_{2} \cdots \bar{j}_{p+1}}=1$.

For the sake of convenience, we assume in the sequel that $\bar{j}_{i}=i$ for $i \in\langle p+1\rangle$. Otherwise, we can adjust the corresponding columns and rows of all matrices in $\mathscr{A}$ by permutation matrices such that the above assumption holds. It is not difficult to see that such a transformation does not change the existence of CWCLFs of $\mathscr{A}$.

If assumption (H3) holds, by using Lemmas 2 and 3, we know that the $p$-dimensional vector $\theta_{12 \cdots p+1}$ is well defined by (14). Construct the $(n-p) \times n$-matrix of the form

$$
B_{k}=\operatorname{diag}\left\{\left(\theta_{12 \cdots p+1}^{T}, 1\right), I_{n-p-1}\right\} A_{k} .
$$

Let

$$
\mathscr{M}_{q}=\left\{B_{q k}: k \in\langle m\rangle\right\},
$$

where $q \in\langle p+1\rangle$ and the $(n-p) \times(n-p)$-matrix $B_{q k}$ has the form

$$
\begin{aligned}
& B_{q k}=\left[\operatorname{col}_{q}\left(B_{k}\right), \operatorname{col}_{p+2}\left(B_{k}\right), \operatorname{col}_{p+3}\left(B_{k}\right), \ldots,\right. \\
& \left.\quad \operatorname{col}_{n}\left(B_{k}\right)\right] .
\end{aligned}
$$


Theorem 9. Assume that (H3) holds. There exists a CWCLF of $\mathscr{A}$ if and only if $\theta_{12 \ldots p+1}>0$, and there exists a CWCLF of $\bigcup_{q \in\langle p+1\rangle} \mathscr{M}_{q}$. In addition, all CWCLFs of $\mathscr{A}$ have the form $\mu\left(\theta_{12 \cdots p+1}^{T}, 1, \phi^{T}\right)^{T}$, where $\mu>0$ is a constant, $\phi>0$ is a $(n-p-1)$-dimensional vector, and $\left(1, \phi^{T}\right)^{T}$ is a CWCLF of $\bigcup_{q \in\langle p+1\rangle} M_{q}$.

Proof.

Necessity. We first get from (H3) and Lemma 3 that $\mathscr{L}_{j_{1} j_{2} \cdots j_{q}}$ has a CLCLF for any SPI $\left\{j_{1}, j_{2}, \ldots, j_{q}\right\}$ and any $q \in\langle p\rangle$. By using (H3) and Lemma 5, all CWCLFs of $\mathscr{L}_{12 \cdots p+1}$ have the form $\mu\left(\theta_{12 \cdots p+1}^{T}, 1\right)^{T}$ with $\mu>0$, and hence $\theta_{12 \cdots p+1}>$ 0 . Assume that $v$ is a CWCLF of $\mathscr{A}$. Then, there exists appropriate $\mu>0$ such that $v=\mu\left(\theta_{12 \cdots p+1}^{T}, 1, \phi^{T}\right)^{T}$, where $\phi$ is the corresponding $(n-p-1)$-dimensional vector. Based on a straightforward computation, it is not difficult to conclude from (33) and (35) that $\left(1, \phi^{T}\right)^{T}$ is a CWCLF of $\bigcup_{q \in\langle p+1\rangle} \mathscr{M}_{q}$.

Sufficiency. Since $\theta_{12 \cdots p+1}>0$, we first have that $\left(\theta_{12 \cdots p+1}^{T}, 1\right)^{T}$ is a CWCLF of $\mathscr{L}_{12 \ldots p+1}$ from (11), (14), and (15). If $\left(1, \phi^{T}\right)^{T}$ is a CWCLF of $\bigcup_{q \in\langle p+1\rangle} \mathscr{M}_{q}$, we can get from (33) and (35) that $\left(\theta_{12 \cdots p+1}^{T}, 1, \phi^{T}\right)^{T}$ is a CWCLF of $\mathscr{A}$ according to a direct computation. This completes the proof of Theorem 9 .

Remark 10. By virtue of Theorem 9, the existence of CWCLFs of $\mathscr{A}$ reduces to the existence of CWCLFs of lower dimensional Metzler matrices.

\section{Numerical Examples}

In this section, we present two examples to illustrate the main results.

Example 1. Consider the family of $3 \times 3$ Metzler matrices $\mathscr{A}=$ $\left\{A_{1}, A_{2}\right\}$ with

$$
\begin{aligned}
& A_{1}=\left(\begin{array}{ccc}
-1 & \frac{1}{3} & \frac{4}{5} \\
1 & -1 & \frac{4}{5} \\
0 & 0 & -1
\end{array}\right), \\
& A_{2}=\left(\begin{array}{ccc}
-1 & 1 & 0 \\
\frac{1}{5} & -1 & \frac{1}{2} \\
\frac{1}{2} & 0 & -1
\end{array}\right) .
\end{aligned}
$$

Since the combination matrix

$$
A_{122}=\left(\begin{array}{ccc}
-1 & 1 & 0 \\
1 & -1 & \frac{1}{2} \\
0 & 0 & -1
\end{array}\right)
$$

has a zero eigenvalue, there is not any CLCLF of $\mathscr{A}$. We now verify whether $\mathscr{A}$ has CWCLF.
Step 1. For the SPI $\{1,2\}$, we have that $\theta_{12}=1$ and $\lambda_{12}=1$.

Step 2. From (33) and (35), a straightforward computation yields that

$$
\begin{aligned}
& \mathscr{M}_{1}=\left\{\left(\begin{array}{cc}
0 & \frac{8}{5} \\
0 & -1
\end{array}\right),\left(\begin{array}{cc}
-\frac{4}{5} & \frac{1}{2} \\
\frac{1}{2} & -1
\end{array}\right)\right\}, \\
& \mathscr{M}_{2}=\left\{\left(\begin{array}{cc}
-\frac{2}{3} & \frac{8}{5} \\
0 & -1
\end{array}\right),\left(\begin{array}{cc}
0 & \frac{1}{2} \\
0 & -1
\end{array}\right)\right\} .
\end{aligned}
$$

Step 3. It is not difficult to see that all CWCLFs of $\mathscr{M}_{1} \cup \mathscr{M}_{2}$ have the form $\mu(1,8 / 5)$ for $\mu>0$.

Therefore, we get from Theorem 9 that all CWCLFs of $\mathscr{A}$ have the form $\mu(1,1,8 / 5)$ for $\mu>0$. Moreover, it is easy to see that there is not a CJCLF of $\mathscr{A}$.

Example 2. Consider the family of $4 \times 4$ Metzler matrices $\mathscr{A}=$ $\left\{A_{1}, A_{2}\right\}$ with

$$
A_{1}=\left(\begin{array}{cccc}
-1 & 0 & \frac{1}{3} & 0 \\
0 & -1 & \frac{1}{3} & 0 \\
0 & 0 & -1 & \frac{1}{4} \\
1 & 1 & 0 & -1
\end{array}\right)
$$

$$
A_{2}=\left(\begin{array}{cccc}
-1 & 0 & \frac{1}{3} & \frac{1}{2} \\
0 & -1 & \frac{1}{3} & \frac{1}{2} \\
0 & 0 & -1 & 0 \\
\frac{1}{3} & \frac{1}{3} & 0 & -1
\end{array}\right) \text {. }
$$

Since the combination matrix

$$
A_{1112}=\left(\begin{array}{cccc}
-1 & 0 & \frac{1}{3} & \frac{1}{2} \\
0 & -1 & \frac{1}{3} & \frac{1}{2} \\
0 & 0 & -1 & 0 \\
1 & 1 & 0 & -1
\end{array}\right)
$$

has a zero eigenvalue, there is not any CLCLF of $\mathscr{A}$. We now verify whether $\mathscr{A}$ has CWCLF.

Step 1. Note that $\lambda_{12}=\lambda_{13}=\lambda_{23}=\lambda_{34}=0$ and $\lambda_{14}=\lambda_{24}=$ $1 / 2$. That is, $\lambda_{j_{1} j_{2}}<1$ for any SPI $\left\{j_{1}, j_{2}\right\}$.

Step 2. For an SPI $\{1,2,4\}$, a straightforward computation yields that $\theta_{124}=(1,1)^{T}$ and $\lambda_{124}=1$. We now adjust the corresponding columns and rows of all matrices in $\mathscr{A}$ 
by permutation matrices such that they take the following form:

$$
\begin{aligned}
& A_{1}=\left(\begin{array}{cccc}
-1 & 0 & 0 & \frac{1}{3} \\
0 & -1 & 0 & \frac{1}{3} \\
1 & 1 & -1 & 0 \\
0 & 0 & \frac{1}{4} & -1
\end{array}\right) \\
& A_{2}=\left(\begin{array}{cccc}
-1 & 0 & \frac{1}{2} & \frac{1}{3} \\
0 & -1 & \frac{1}{2} & \frac{1}{3} \\
\frac{1}{3} & \frac{1}{3} & -1 & 0 \\
0 & 0 & 0 & -1
\end{array}\right) .
\end{aligned}
$$

Step 3. According to a direct computation, we get from (33) and (35) that

$$
\begin{aligned}
& \mathscr{M}_{1}=\mathscr{M}_{2}=\left\{\left(\begin{array}{cc}
0 & \frac{2}{3} \\
0 & -1
\end{array}\right),\left(\begin{array}{cc}
-\frac{2}{3} & \frac{2}{3} \\
0 & -1
\end{array}\right)\right\}, \\
& \mathscr{M}_{3}=\left\{\left(\begin{array}{cc}
-1 & \frac{2}{3} \\
\frac{1}{4} & -1
\end{array}\right),\left(\begin{array}{cc}
0 & \frac{2}{3} \\
0 & -1
\end{array}\right)\right\} .
\end{aligned}
$$

Step 4. It can be seen that all CWCLFs of $\mathscr{M}_{1} \cup \mathscr{M}_{2} \cup \mathscr{M}_{3}$ have the form $a(1, b)^{T}$ for $a>0$ and $2 / 3 \leq b \leq 4$.

Therefore, we get from Theorem 9 that all CWCLFs of $\mathscr{A}$ have the form $a(1,1, b, 1)^{T}$ with $a>0$ and $2 / 3 \leq b \leq 4$. In addition, it is easy to verify that $(1,1,1,1)^{T}$ is a CJCLF of $\mathscr{A}$.

\section{Conclusion}

The existence of a class of CWCLFs has been investigated in this paper, which generalize the usual CLCLFs and can be applied to stability analysis of positive switched linear systems. By using matrix theory, necessary and sufficient conditions for the existence of CWCLFs have been established. Moreover, the algebraic structure of all CWCLFs is described clearly. Two numerical examples are also given to illustrate the effectiveness of the obtained results.

\section{Conflicts of Interest}

The authors declare that they have no conflicts of interest.

\section{Acknowledgments}

This work is supported by the National Natural Science Foundation of China (Grants nos. 61473133, 11671227, and 61374074) and the Natural Science Foundation of Shandong Province (Grant no. JQ201119).

\section{References}

[1] L. Farina and S. Rinaldi, Positive Linear Systems: Theory and Applications, Wiley-Interscience, New York, NY, USA, 2000.

[2] N. Zhang, Y. Sun, and P. Zhao, "State bounding for homogeneous positive systems of degree one with time-varying delay and exogenous input," Journal of The Franklin Institute, vol. 354, no. 7, pp. 2893-2904, 2017.

[3] Y. Sun and F. Meng, "Reachable set estimation for a class of nonlinear time-varying systems," Complexity, vol. 2017, Article ID 5876371, 6 pages, 2017.

[4] Y. Sun and L. Wang, "Ho consensus of second-order multiagent systems with asymmetric delays," Systems \& Control Letters, vol. 61, no. 8, pp. 857-862, 2012.

[5] Y. Cao and Y. Sun, "Consensus of discrete-time third-order multi-agent systems in directed networks," Neurocomputing, vol. 177, pp. 394-400, 2016.

[6] Y. Cao, Y. Sun, and X.-J. Xie, "Explicit condition for consensus of third-order discrete-time multi-agent systems without accelerated velocity measurements," Journal of The Franklin Institute, vol. 354, no. 12, pp. 5056-5066, 2017.

[7] Z. Ji and H. Yu, "A new perspective to graphical characterizition of multi-agent controllability," IEEE Trans. Cybern, vol. 47, pp. 1471-1483, 2017.

[8] F. Xiao, T. Chen, and H. Gao, "Consensus in time-delayed multiagent systems with quantized dwell times," Systems \& Control Letters, vol. 104, pp. 59-65, 2017.

[9] L. Fainshil, M. Margaliot, and P. Chigansky, "On the stability of positive linear switched systems under arbitrary switching laws," Institute of Electrical and Electronics Engineers Transactions on Automatic Control, vol. 54, no. 4, pp. 897-899, 2009.

[10] X. Liu, "Stability analysis of switched positive systems: A switched linear copositive Lyapunov function method," IEEE Transactions on Circuits and Systems II: Express Briefs, vol. 56, no. 5, pp. 414-418, 2009.

[11] H. Alonso and P. Rocha, "A general stability test for switched positive systems based on a multidimensional system analysis," Institute of Electrical and Electronics Engineers Transactions on Automatic Control, vol. 55, no. 11, pp. 2660-2664, 2010.

[12] X. Liu and C. Dang, "Stability analysis of positive switched linear systems with delays," Institute of Electrical and Electronics Engineers Transactions on Automatic Control, vol. 56, no. 7, pp. 1684-1690, 2011.

[13] X. Zhao, L. Zhang, P. Shi, and M. Liu, "Stability of switched positive linear systems with average dwell time switching," Automatica, vol. 48, no. 6, pp. 1132-1137, 2012.

[14] X. Zhao, X. Liu, S. Yin, and H. Li, "Improved results on stability of continuous-time switched positive linear systems," Automatica, vol. 50, no. 2, pp. 614-621, 2014.

[15] Y. Li, Y. Sun, and F. Meng, "New criteria for exponential stability of switched time-varying systems with delays and nonlinear disturbances," Nonlinear Analysis: Hybrid Systems, vol. 26, pp. 284-291, 2017.

[16] D. Tian and S. Liu, "Exponential stability of switched positive homogeneous systems," Complexity, vol. 2017, Article ID 4326028, 8 pages, 2017.

[17] Y. Li, Y. Sun, F. Meng, and Y. Tian, "Exponential stabilization of switched time-varying systems with delays and disturbances," Applied Mathematics and Computation, vol. 324, pp. 131-140, 2018. 
[18] O. Mason and R. Shorten, "On linear copositive Lyapunov functions and the stability of switched positive linear systems," Institute of Electrical and Electronics Engineers Transactions on Automatic Control, vol. 52, no. 7, pp. 1346-1349, 2007.

[19] F. Knorn, O. Mason, and R. Shorten, "On linear co-positive Lyapunov functions for sets of linear positive systems," Automatica, vol. 45, no. 8, pp. 1943-1947, 2009.

[20] E. Fornasini and M. E. Valcher, "Linear copositive Lyapunov functions for continuous-time positive switched systems," Institute of Electrical and Electronics Engineers Transactions on Automatic Control, vol. 55, no. 8, pp. 1933-1937, 2010.

[21] Y. Sun and $\mathrm{Z}$. Wu, "On the existence of linear copositive Lyapunov functions for 3-dimensional switched positive linear systems," Journal of The Franklin Institute, vol. 350, no. 6, pp. 1379-1387, 2013.

[22] Z. Wu and Y. Sun, "On easily verifiable conditions for the existence of common linear copositive Lyapunov functions," Institute of Electrical and Electronics Engineers Transactions on Automatic Control, vol. 58, no. 7, pp. 1862-1865, 2013.

[23] D. Cheng, J. Wang, and X. Hu, "An extension of LaSalle's invariance principle and its application to multi-agent consensus," Institute of Electrical and Electronics Engineers Transactions on Automatic Control, vol. 53, no. 7, pp. 1765-1770, 2008.

[24] J. Wang, D. Cheng, and X. Hu, "An extension of LaSalle's invariance principle for a class of switched linear systems," Systems \& Control Letters, vol. 58, no. 10-11, pp. 754-758, 2009.

[25] Y. Sun, "Stability analysis of positive switched systems via joint linear copositive Lyapunov functions," Nonlinear Analysis: Hybrid Systems, vol. 19, pp. 146-152, 2016.

[26] Y. Sun, Y. Tian, and -J. Xie, "Stabilization of positive switched linear systems and its application in consensus of multi-agent systems," IEEE Xplore: IEEE Transactions on Automatic Control, vol. 62, pp. 6608-6613, 2017.

[27] Z. Wu and Y. Sun, "Common joint linear copositive Lyapunov functions for positive switched systems," Journal of The Franklin Institute, vol. 351, no. 5, pp. 2504-2514, 2014.

[28] K. S. Narendra and R. Shorten, "Hurwitz stability of Metzler matrices," Institute of Electrical and Electronics Engineers Transactions on Automatic Control, vol. 55, no. 6, pp. 1484-1487, 2010. 


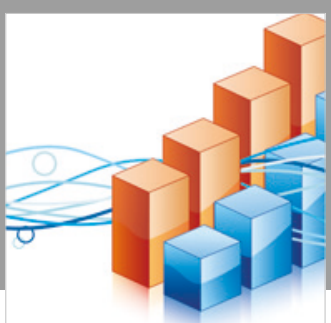

Advances in

Operations Research

\section{-n-m}
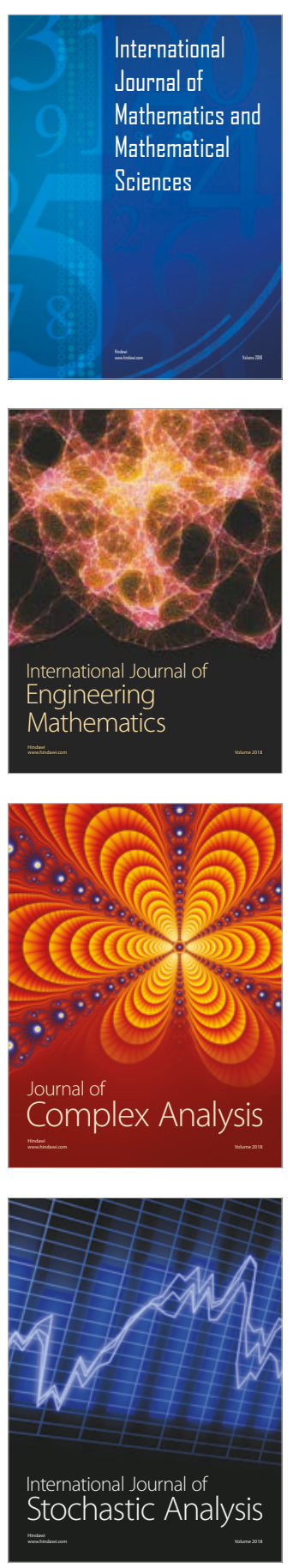
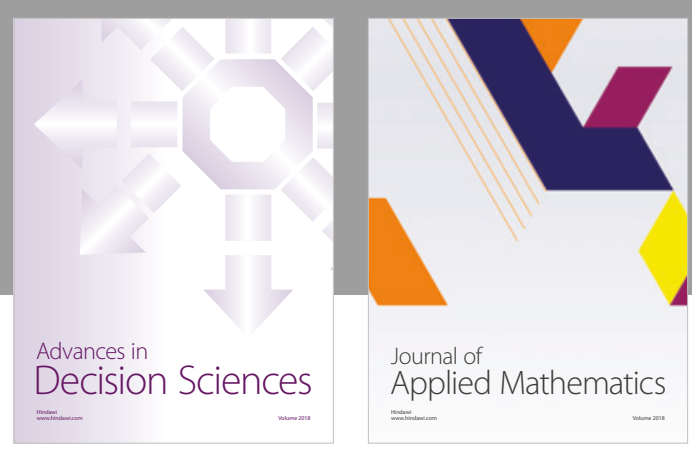

Journal of

Applied Mathematics
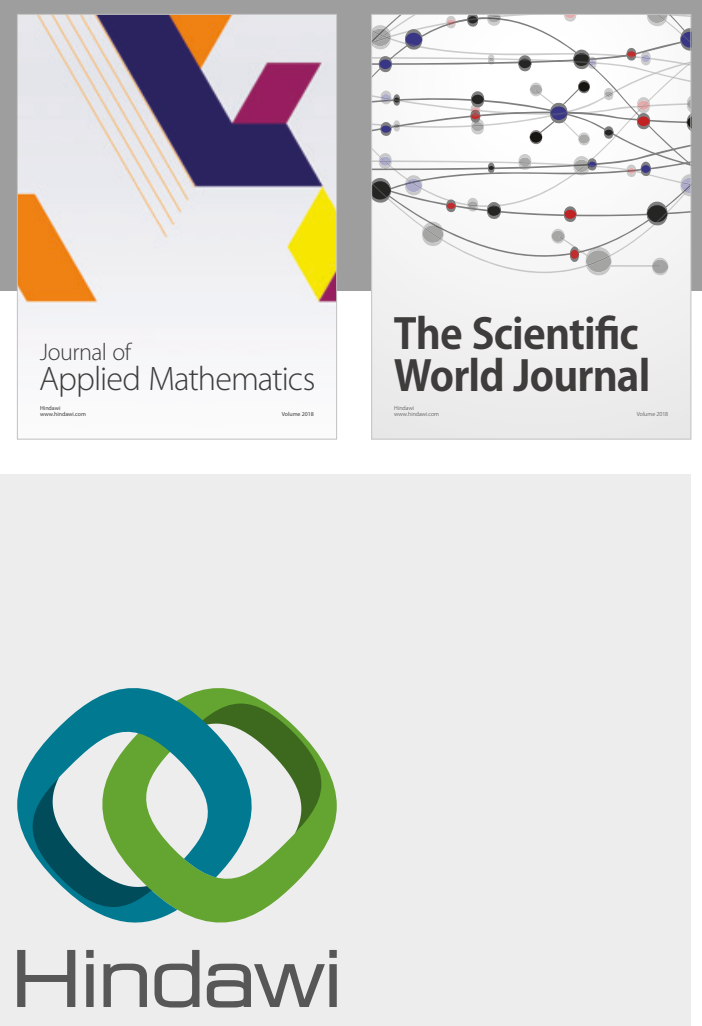

Submit your manuscripts at

www.hindawi.com

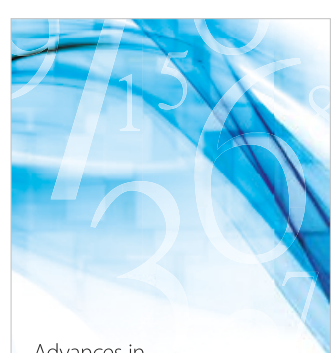

Advances in
Numerical Analysis
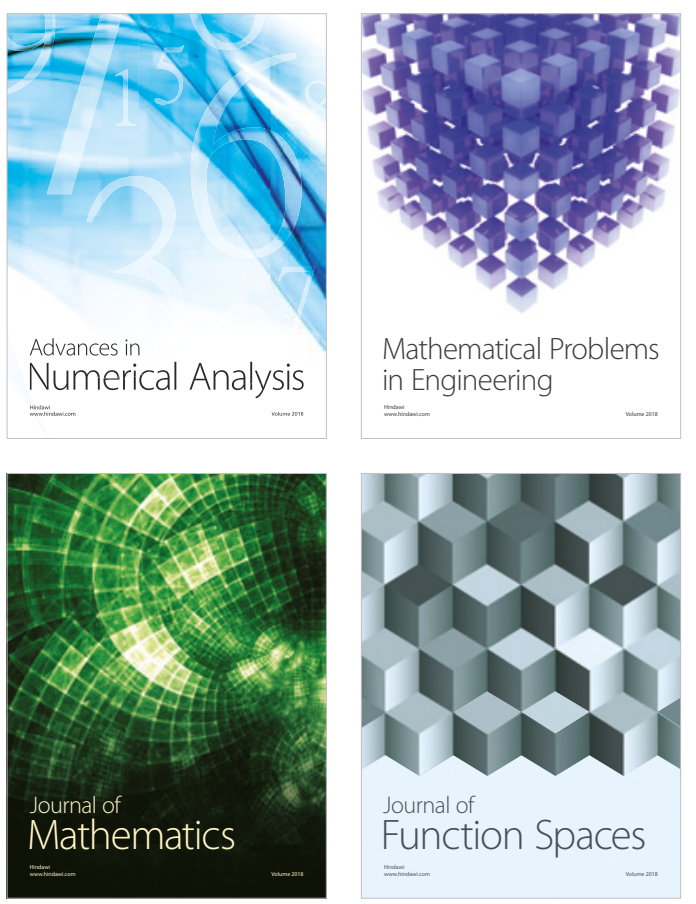

Mathematical Problems in Engineering

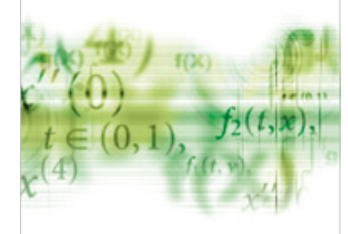

International Journal of

Differential Equations

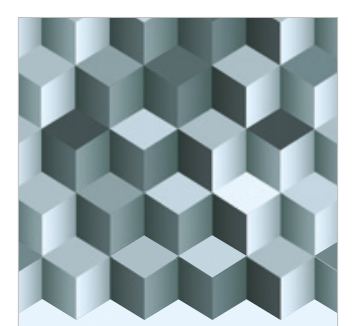

Journal of

Function Spaces
The Scientific

World Journal

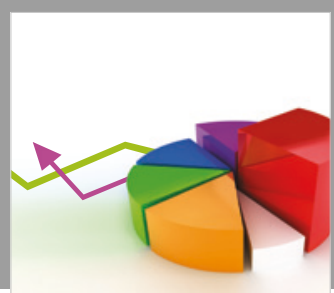

Journal of

Probability and Statistics
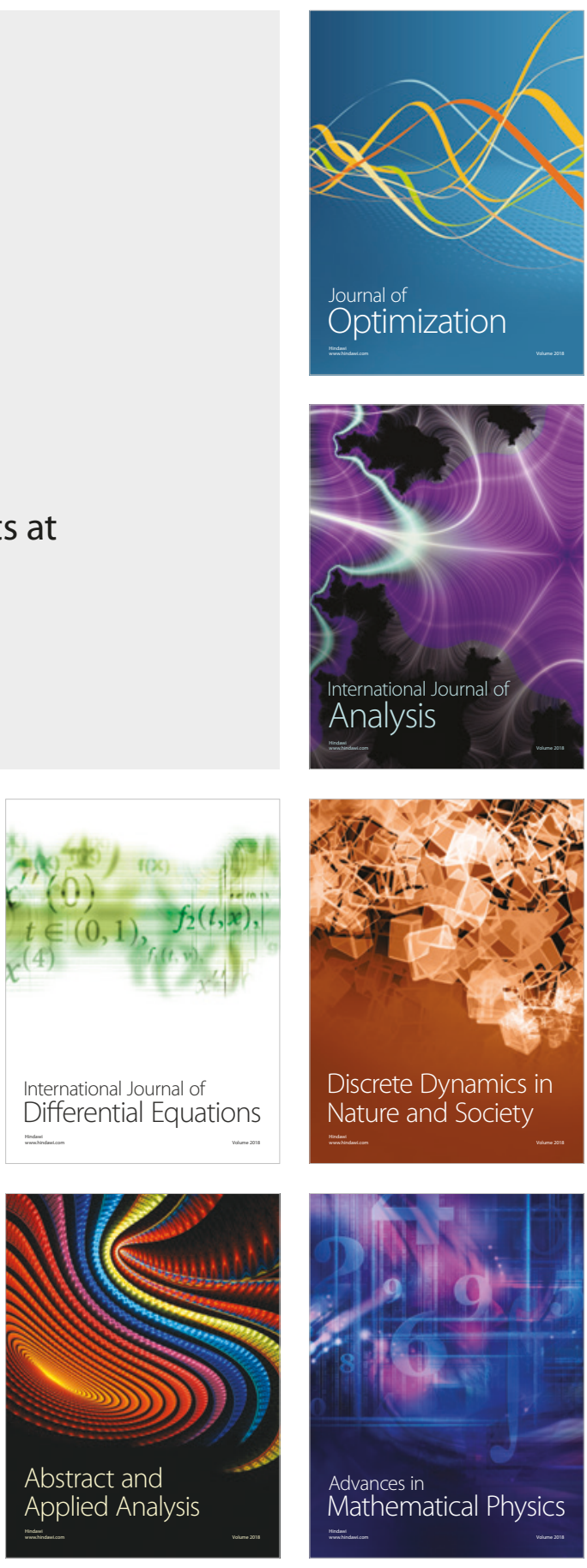